\title{
Free-Floating Thrombi in the Right Atrium Causing Pulmonary Embolism
}

\author{
Amr Abdin \\ Cardiology Department, Bayreuth Hospital ,Bayreuth, Germany
}

\begin{abstract}
A 74-year-old man presented to our Emergency Department with acute dyspnoea. His electrocardiogram showed atrial flutter with 2:1 block and a rate of $150 \mathrm{bpm}$. Initial investigations revealed a D-dimer level of $6.01 \mathrm{mg} / \mathrm{dl}$. Based on the patient's complaints and the high D-dimer level, computed tomography pulmonary angiography was immediately performed. This showed no evidence of pulmonary embolism, but there were pneumatic changes in the right upper lung lobe. Antibiotics treatment was started with pipracillin/tazobactam, after which the patient's condition improved. However, on the third day after admission he developed acute dyspnoea, diaphoresis and cardiopulmonary instability immediately after defecation. To promptly confirm our clinical suspicion of pulmonary embolism, a transthoracic echocardiography was carried out. This demonstrated a worm-like, mobile mass in the right heart. The right ventricle was enlarged, and paradoxical septal motion was present, indicating right ventricular pressure overload. The systolic tricuspid valvular gradient was $56 \mathrm{mmHg}$. The patient was treated with thrombolysis. His condition was greatly clinically improved after 3 hours. After 10 days of hospitalization, the patient was discharged.
\end{abstract}

Keywords: Embolism, echocardiography, thrombus, thrombolysis

Received: $19 / 07 / 2014$

Accepted: $16 / 10 / 2014$

Published: $11 / 11 / 2014$

How to cite this article: Abdin A. Free-Floating Thrombi in the Right Atrium Causing Pulmonary Embolism. EJCRIM 2014;1:doi:

10.12890/2014_000108

Conflicts of Interests: The authors declare that they have no conflicts of interest related to this research.

\section{Case presentation}

A 74-year-old man, with no cardiovascular history, presented to our Emergency Department with acute dyspnoea. His echocardiogram (ECG) showed atrial flutter with 2:1 block and a rate of $150 \mathrm{bpm}$ (Fig. 1). Based on the patient's complaints and a D-dimer level of $6.01 \mathrm{mg} / \mathrm{dl}$, a computed tomography (CT) pulmonary angiography was immediately performed. This showed no evidence of pulmonary embolism, but there were pneumatic changes in the right upper lung lobe. Antibiotics treatment was started with pipracillin/tazobactam. The patient was admitted to our cardiology ward and was also treated with unfractionated heparin. 


\section{European Journal}

of Case Reports in

Internal Medicine

After this treatment, there was a clear improvement in the patient's condition. However, on the third day after admission he developed acute dyspnoea, diaphoresis and cardiopulmonary instability (blood pressure 80/60, P: 120/m) immediately after passing a stool. The ECG demonstrated sinus tachycardia and T-wave inversion in leads III and aVF. Since the patient had borderline renal function, we did not wish to subject the patient to a second CT scan, so we performed a transthoracic echocardiography to confirm our clinical suspicion of a pulmonary embolism (Figure 1). This demonstrated a worm-like, mobile mass in the right heart. The right ventricle was enlarged, and paradoxical septal motion was present, indicating right ventricular pressure overload. The systolic tricuspid valvular gradient was 56 $\mathrm{mmHg}$.

The patient was treated with thrombolysis and his clinical condition greatly improved within 3 hours. Serial echocardiograms demonstrated complete dissolution of the right-sided thrombi. Later, a venous ultrasonography demonstrated that the thrombi originated in the popliteal region of both legs. After 10 days of hospitalization, the patient was discharged.
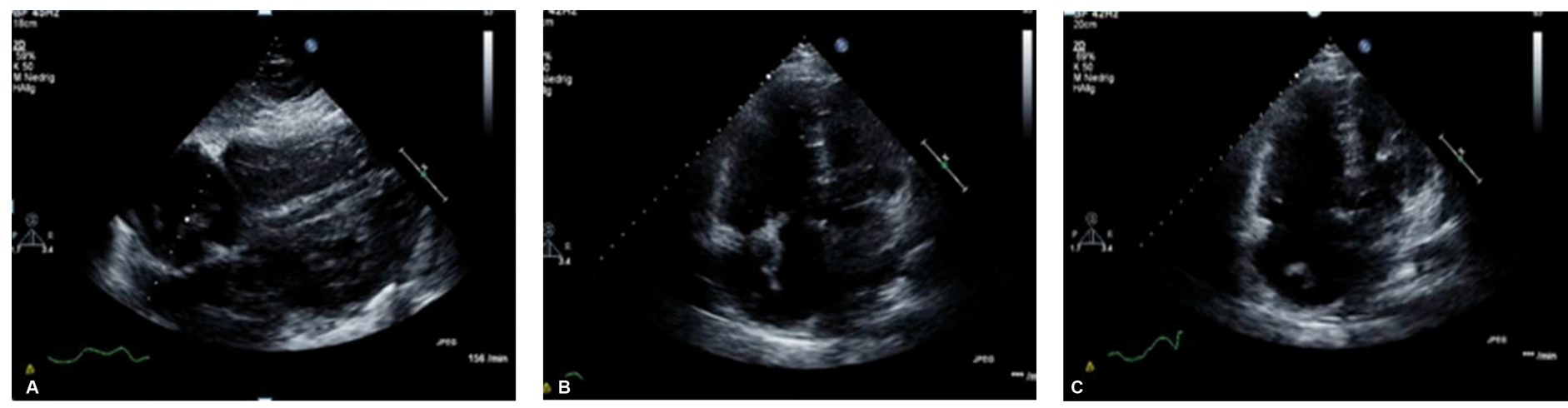

Figure 1 a-c: Transthoracic Echocardiography demonstrated a worm-like, mobile mass in the right heart.

\section{Learning Points}

- A prompt transthoracic echocardiogram can be highly informative in patients with suspected pulmonary embolism.

- Free-floating right heart thrombi are a rare phenomenon. Serial echocardiographic examinations are useful when the clinical status deteriorates, because they may demonstrate a thrombus that was not detected on the initial examination. ${ }^{1}$

- Right heart thrombi-in-transit and deep venous thrombus should be sought in patients with massive pulmonary embolism. Echocardiography is necessary to assess the presence of PFO since the therapeutic options may vary in patients with right heart thrombus. ${ }^{1}$

- Thrombolysis is a simple and fast treatment option with numerous advantages including acceleration of pulmonary reperfusion; reduction in pulmonary hypertension; improvement of right ventricular function; and the possibility of dissolving the intracardiac thrombus, pulmonary embolism and the venous thromboembolism at the same time. ${ }^{1}$ 
European Journal

of Case Reports in

Internal Medicine

\section{References}

1. Chartier L, Béra J, Delomez M, Asseman P, Beregi JP, Bauchart JJ, Warembourg H, Théry C. Free-floating thrombi in the right heart: diagnosis, management, and prognostic indexes in 38 consecutive patients. Circulation 1999;99:2779-2783. 